\title{
First Description of Factors Influencing the Outcome of Developmental Dysplasia of the Hip in Children's Medical Center
}

\author{
Mehrzad Mehdizadeh ${ }^{1}$, Nasir Babakhan Kondori ${ }^{2,}{ }^{*}$, Afrooz Zandifar ${ }^{1}$ and Hooman Alizadeh ${ }^{1}$ \\ ${ }^{1}$ Department of Radiology, Children's Medical Center of Excellence, Tehran University of Medical Sciences, Tehran, Iran \\ ${ }^{2}$ Department of Radiology, Namazi Hospital, Shiraz University of Medical Sciences, Shiraz, Iran \\ "Corresponding author: Department of Radiology, Namazi Hospital, Shiraz University of Medical Sciences, Shiraz, Iran. Tel: +98-9122809709, Email: nasirbabakhan@gmail.com \\ Received 2019 July 10; Revised 2020 March 25; Accepted 2020 May 02.
}

\begin{abstract}
Background: There are several factors may have an impact on the prognosis of developmental dysplasia of the hip (DDH). They may change the outcome and treatment if they are present in a patient.

Objectives: The objective of this study is to determine the association of those factors with the outcome.

Patients and Methods: The study was performed on 74 infants with DDH in Children's Medical Centre. After obtaining informed consent and ethical approval, patients with DDH confirmed by ultrasound were included, and possible risk factors including severity of DDH based on the Graf criteria, follow-up length, age, gender, laterality, type of delivery, amniotic fluid index, fetal anomalies, birth order of children, and methods of treatment were collected and compared against treatment response.

Results: There were 58 female and 19 male patients with a mean age of 6.54 weeks. Bilateral DDH was found in 31 females and nine males. The average alpha and beta angles were $53.19 \pm 5.7$ and $66.53 \pm 6.6$ degree, respectively. The severity of DDH had a significant association with treatment response $(\mathrm{P}=0.003)$. Recovery was better in females than in males $(\mathrm{P}=0.031)$. In addition, the first-born infants had a better response $(\mathrm{P}=0.001)$. A meaningful connection $(\mathrm{P}=0.01)$ was noted between the amniotic fluid index as well as renal/limb anomalies and treatment response. Type of delivery (cesarean versus vaginal) or fetal presentation had no significant association with treatment response $(\mathrm{P}>0.05)$.

Conclusion: Factors with a negative impact on DDH outcomes include the severity of DDH, male gender, higher birth order, oligohydramnios, renal and limb anomalies.
\end{abstract}

Keywords: Newborn, Perinatal Risk Factors, Screening, Ultrasound, Developmental Dysplasia of the Hip

\section{Background}

The frequency of developmental dysplasia of the hip (DDH) is 0.8 - 1 per 1000 births (1). If it is not detected and not corrected in early infancy, it can interfere with normal development and leads to anatomical and functional disorders in adulthood (2). Although there is no definite etiology for DDH, a group of genetic and environmental factors including ligament laxity, breech presentation, postnatal hip joint positions like swaddling, female gender, first delivery, and family history may play a role $(3,4)$.

Physical examinations (Ortolani, Barlow, Galeazzi or Allis) only have $28.1 \%$ sensitivity for DDH (5). Therefore, ultrasound for earlier diagnosis and X-ray for older children should be performed. The treatment aim is to maintain good contact between the femoral head and acetabulum. For this purpose, flexion and abduction in a physiological position are recommended. For older children, how- ever, closed/open reductions may be considered based on their age and conditions (6-8). The severity of DDH and treatment are determined based on the Graf criteria (Table 1). Type 1 does not need treatment. Type 2 a should be observed. The Pavlik harness is the treatment of choice for types 2b, 2c, D, 3 and 4 . However, open reduction sometimes is required for older babies.

There are still some controversies regarding risk factors and whether they ever have any impact on the outcome.

\section{Objectives}

The current study aimed at determining possible risk factors and their effects on treatment response. Good knowledge about these factors could predict DDH outcome and may change the threshold in which a surgeon 


\begin{tabular}{|c|c|c|c|c|c|c|c|}
\hline Type & Maturity & Bony roof & $\begin{array}{c}\alpha \text { Angle (Bony } \\
\text { angle), }\end{array}$ & Bony rim & Cartilage root & $\beta$ Angle, ${ }^{\circ}$ & Age \\
\hline Type I & Mature & Good & $\geq 60$ & Sharp & $\begin{array}{l}\text { Good coverage } \\
\text { femoral head }\end{array}$ & $\mathrm{Ia}=\beta \leq \underset{55}{55 ; \mathrm{Ib}=\beta>}$ & All \\
\hline Type II a+ & $\begin{array}{l}\text { Immature but } \\
\text { appropriate for age }\end{array}$ & Adequate & $50-59$ & Blunt & $\begin{array}{l}\text { Coverage femoral } \\
\text { head }\end{array}$ & & $<3 \mathrm{mo}$ \\
\hline Type II a- & $\begin{array}{l}\text { Immature and } \\
\text { inappropriate for age }\end{array}$ & Deficient & $50-59$ & Rounded & $\begin{array}{l}\text { Coverage femoral } \\
\text { head }\end{array}$ & & $<3 \mathrm{mo}$ \\
\hline Type II b & Delay in development & Deficient & $50-59$ & Rounded & $\begin{array}{l}\text { Coverage femoral } \\
\text { head }\end{array}$ & & $>3 \mathrm{mo}$ \\
\hline Type II c & Stable or unstable & Severely deficient & $43-49$ & Rounded /flat & $\begin{array}{l}\text { Still coverage femoral } \\
\text { head }\end{array}$ & $\beta<77$ & All \\
\hline Type D & Decentering hip & Severely deficient & $43-49$ & Rounded /flat & Displaced & $\beta>77$ & All \\
\hline Type III & Eccentric hip & Poor & $<43$ & Flat & $\begin{array}{l}\text { Labrum pressed } \\
\text { upwards }\end{array}$ & & All \\
\hline Type IV & Eccentric hip & Poor & $<43$ & Flat & $\begin{array}{l}\text { Labrum pressed } \\
\text { downwards }\end{array}$ & & All \\
\hline
\end{tabular}

Abbreviation: DDH, developmental dysplasia of the hip.

may shift toward a more aggressive treatment in earlier stages.

\section{Patients and Methods}

The current descriptive- analytical study was conducted in Tehran, Iran from November 2014 to June 2017. Parents were informed of the details of the study, and informed consent was obtained. Ethical approval was obtained by the ethical committee of this center. The sample size was calculated by the Pukak equation, considering a $95 \%$ confidence level, $80 \%$ strength, $5 \%$ error, and 3 per 1000 frequency. Eighty-four patients out of 399 patients who were referred for hip ultrasound either had DDH or type 2a Graf ultrasound. All of them were younger than 18 weeks. Those who did not attend follow-up ultrasound or those with teratologic dislocation, i.e., myelomeningocele, arthrogryposis, chromosomal abnormalities, lumbosacral disorders, or neuromuscular dislocations, were excluded. In the end, 74 patients were included (Figure 1).

Data were collected using hip ultrasound and questionnaires. The questionnaire contained information about family members, birth order, age, gender, type of delivery, presentation, family history of DDH, and associated

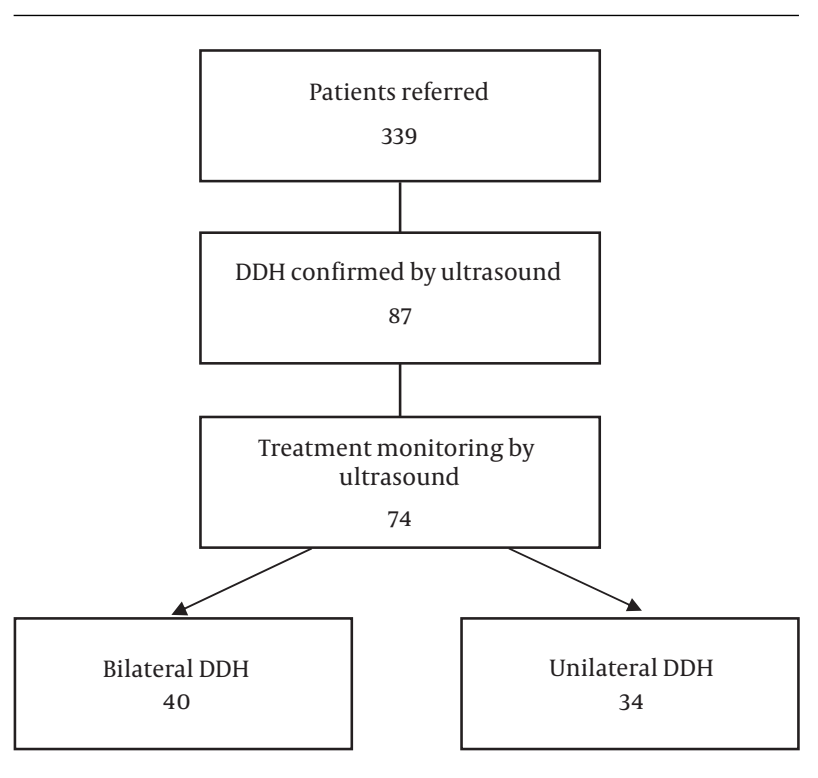

Figure 1. Study population in our survey

anomalies including club foot, torticollis, and meningocele. An ultrasound exam was performed after two weeks from the onset of treatment. In some cases, further followup ultrasound exams were done every four weeks, and 
treatment response was assessed based on ultrasound morphology, alpha and beta angles. We used a 5-MHz linear probe of an ultrasound machine (50-G Siemens, Erlangen, Germany) to obtain coronal views in lateral decubitus. The alpha and beta angles were measured.

Statistical analysis was performed using SPSS version 18 (SPSS Inc. Released 2009. PASW Statistics for Windows, Version 18.0. Chicago: SPSS Inc.). The results were expressed as mean \pm standard deviation (SD) for quantitative, and as frequency and percentage for qualitative data. Patients were divided into two groups: the responsive group who had normal ultrasound report of Graf type 1 at the end of treatment and the non-responsive group who did not show complete recovery to type $1 \mathrm{Graf}$. The relationship between the treatment response and study variables including gender, age, family history, presentation, type of delivery, and associated anomalies were measured using the chi-square test for qualitative variables and $t$-test for quantitative ones. To assess the predictor factors on treatment response, simple and multiple logistic regression were used. To evaluate the relationship between predictor variables (including gender, gestational age at birth, type of delivery, fetal presentation, amniotic fluid volume, order of children, family history of DDH, limb anomalies and renal anomalies) and treatment response, crude and adjusted odds ratio (OR) and 95\% confidence Interval (CI) were used. In all items, results were considered statistically significant if P value was less than 0.05.

\section{Results}

The current study included 58 female and 19 male infants with a mean age of 6.54 weeks. Bilateral DDH was observed in 31 female and nine male patients. All infants were monitored for 3 - 38 weeks (average 6.4). Two infants had just one follow-up ultrasound, 52 patients had two ultrasounds, and the rest underwent 3 - 7 ultrasound exams (Figures 2-5). From all 114 hip joints, 63 (55.3\%) hips were left-sided, and 51 (44.7\%) were right-sided. Mean alpha and beta angles were $53.18 \pm 5.7$ (range: 39 - 59) and $66.53 \pm 6.6$ (range: 53 - 83) degrees, respectively. Graf type of involvement was 2a+ in 47 hips, 2a- in 19 hips, 2 b in eight hips, 2c in seven hips, type D in 12 hips, type 3 in 13 hips, and the remaining eight hip joints had type 4.

All hip joints with type $2 \mathrm{a}$ involvement were just observed. Treatment by medical brace was done for 19 hips with type 2a. The Pavlik harness orthosis was applied for 15 hips including eight hips with type $2 \mathrm{~b}$ and seven hips with type 2c. Closed reduction and the Pavlik harness orthosis were applied for 29 joints including 12 hips with type D, 11 hips with type 3 , and six hips with type 4 . Open reduction was performed for four joints including two type 3 hips and two type 4 hips. Recovery was observed in 98 involved hips (86\%), however, 16 joints did not show improvement and required further treatment. Among the subjects, 55 infants were born by cesarean section and the other 19 by vaginal delivery. The cephalic presentation was observed in 68 subjects and breech presentation in the other six patients. Oligohydramnios was observed in four cases; while, 70 patients were reported normal during pregnancy. Fortyfour patients were first child, nine patients had a positive family history, and nine patients had limb anomalies. Musculoskeletal anomalies include: clubfoot (8.8\%), patella dislocation (0.9\%), scoliosis (1.8\%), radial dysgenesis (0.9\%), and hydronephrosis (2.6\%).

A better response was achieved in patients with type $2 \mathrm{a}$ and those with milder DDH based on Graf classification. For instance, $8.3 \%$ of type D, $53.8 \%$ of type 3 , and all of the type 4 joints did not respond to treatment. Likewise, the mean alpha angle in the non-responsive group was 43.25 \pm 3.2 degree in contrast with $54.02 \pm 5$ in the responsive group, which was significantly lower $(P=0.006)$. Similarly, the mean beta angle was $80 \pm 7.1$ degrees in the nonresponsive group compared with $66.38 \pm 5.6$ in the responsive group, which was significantly higher $(\mathrm{P}=0.034)$.

Of 114 affected hip joints, 89 hips (78\%) were observed in female patients and the other $25(22 \%)$ in male ones. Besides, $89.8 \%$ of affected hips in females and $72 \%$ in male patients showed complete recovery. Overall, $81.6 \%$ of treated cases were females and $18.4 \%$ were males. These results showed that response to treatment was better in females than males $(\mathrm{P}=0.031)$. Being female increases the treatment response by a factor of 8.21 .

Five $(4.4 \%)$ patients had oligohydramnios history, only two of which (40\%) had a good response during follow-up. The amniotic fluid had a significant correlation with response to treatment $(\mathrm{P}=0.013)$. A normal amniotic fluid increases the treatment response by a factor of 16.92 .

Among first-born children, 93.8\% responded to treatment, the second-born children showed an $80.9 \%$ response rate, and the third- and next-born children showed only $42.85 \%$ recovery. Overall, $62.2 \%$ of the patients were firstborn children, $34.7 \%$ second-born, and 3.1\% next-born children. Treatment response was significantly better among first-born children $(\mathrm{P}=0.001)$.

Treatment was also better (91\%) in those without limb anomalies compared with those with anomalies (50\%) (P $=0.001$, and a significant connection between the pres- 


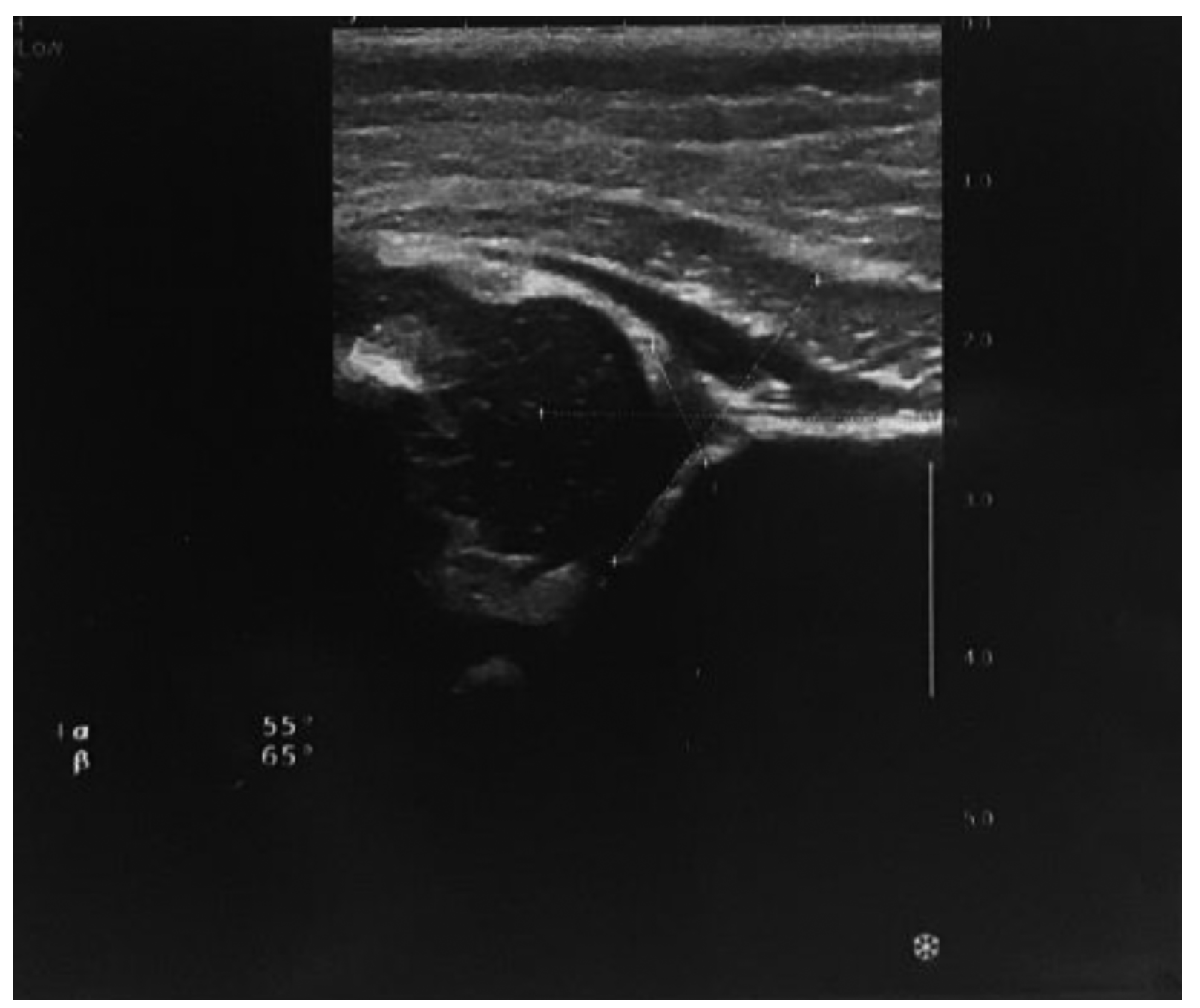

Figure 2. Five-week infant with type II a+ developmental dysplasia of the hip (DDH). Ultrasound measures: alpha 55 and beta 65 degree.

ence of anomalies and failure to response $(P=0.002)$ was noted. Among limb anomalies, the ones associated with poor response were radial dysgenesis, scoliosis, and clubfoot in the order of frequency. Of the patients with renal anomalies, no one showed improvement, while in patients without renal problems, the response rate was $88.2 \%$. Of all patients in the current study, $12.3 \%$ had limb anomalies. The average follow-up time in patients with anomalies was 9.6 weeks, and 8.6 weeks in patients without anomalies. Among the infants with limb anomalies, the ones with patellar dislocation had the longest follow-up, 22 weeks, while in scoliosis and clubfoot it was 3 weeks and 10.63 weeks, respectively. Fifty percent of patients with anomaly showed good response compared with a $91 \%$ response rate in the group without anomaly. Also, $43.8 \%$ of patients in the non-responsive group belonged to the anomaly group. Having no anomaly increases the treatment response by a factor of 5.02. Therefore, the presence of limb anomalies significantly decreased treatment response.

There was no significant correlation between the length of follow-up and response rate. $(\mathrm{P}=0.30)$ Infants with good response attended more in the follow-up sessions. $(\mathrm{P}=0.001)$ All patients that attended five sessions or more showed complete recovery. Twenty-five percent of cases that required further treatment due to their poor response, had only one and 50\% of them had two followup sessions. Results also showed that gestational age at birth, birth weight, sidedness, laterality, type of delivery (cesarean versus vaginal), fetal presentation, and positive family history were not significantly connected with re- 


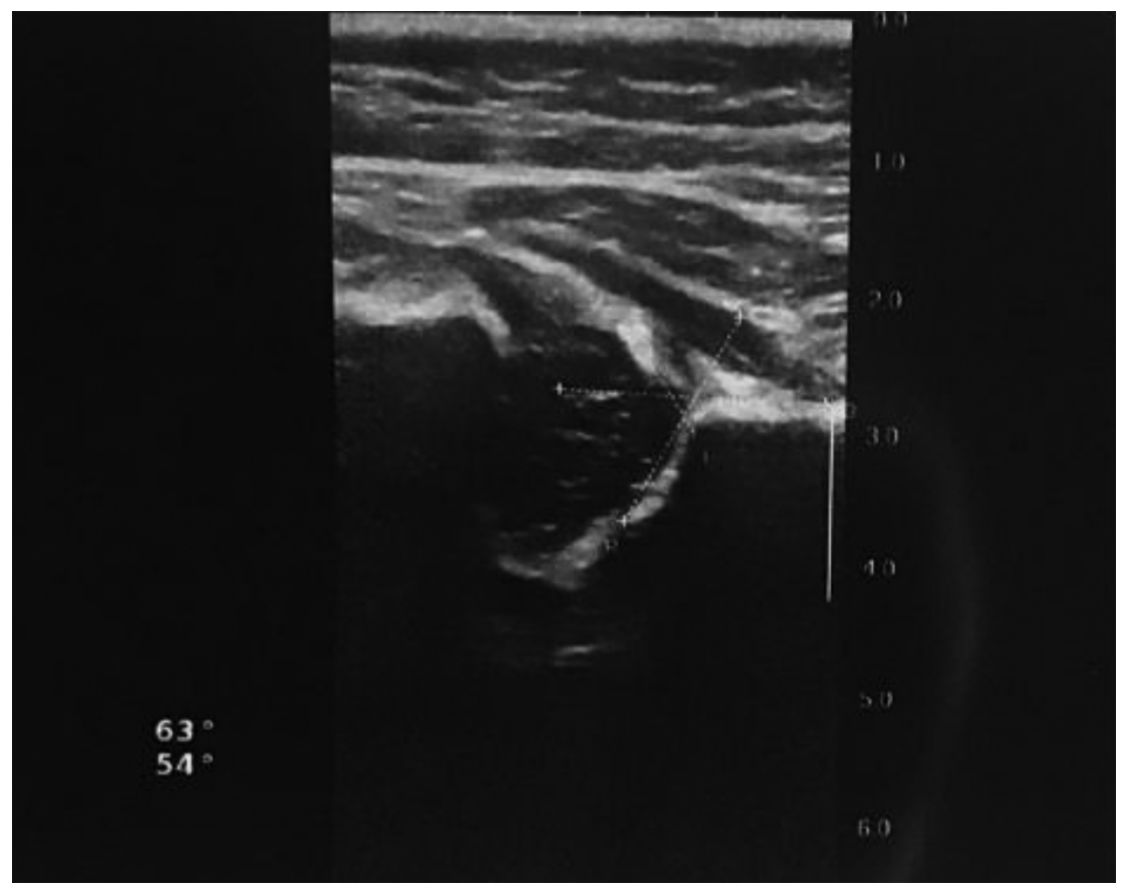

Figure 3. Nine-week infant. The same patients in Figures 1 after 4 weeks shows complete recovery to type 1 (alpha $=63$ and beta $=54$ ).

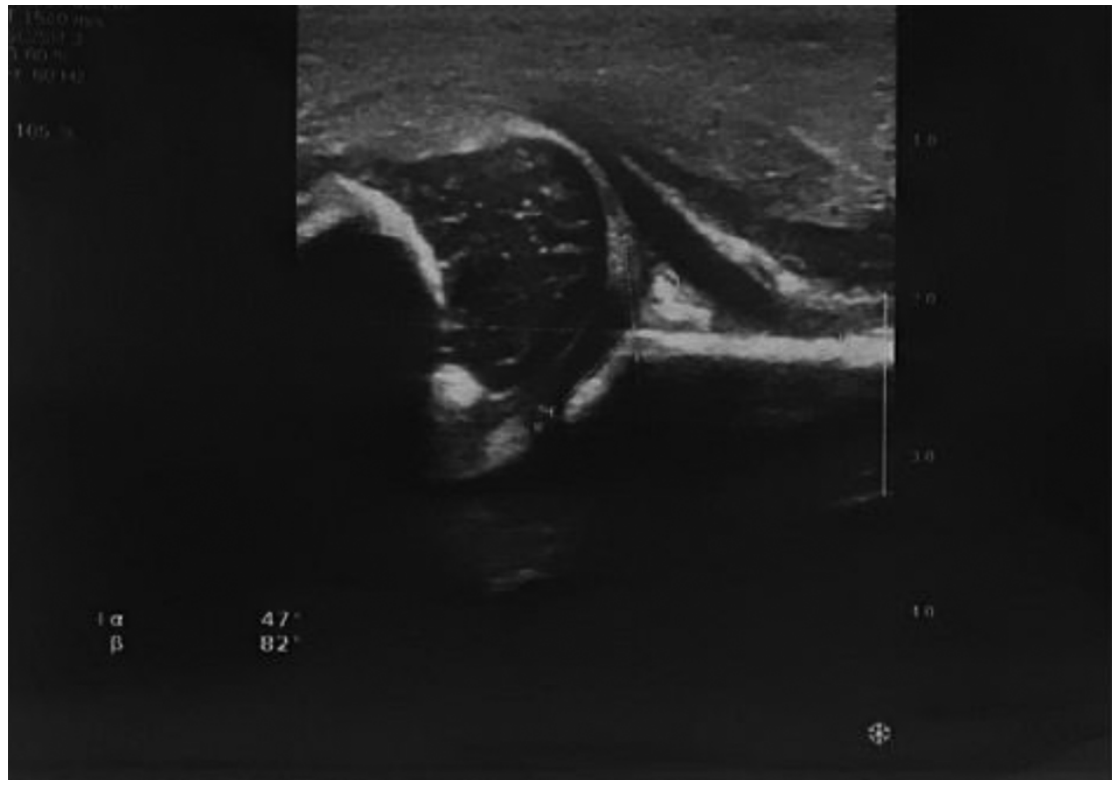

Figure 4. Three-week infant with type D developmental dysplasia of the hip (DDH). Ultrasound measures: alpha 47 and beta 82 degree.

sponse to treatment. Natural delivery decreases the treatment response only by a factor of 0.12. Left hip involvement with $55.3 \%$ (63 joints) was more common than right side involvement. Of those with good response, $56.1 \%$ ( 64 joints) had left side involvement and 43.9\% (50 joints) had right side involvement. However, the difference was not significant (Table 2). 
Table 2. Simple and Multiple Logistic Regression Analysis for Evaluation of Effects of Predictor Variables on Treatment Response of DDH

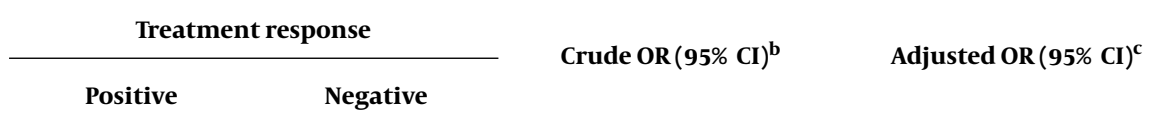

\begin{tabular}{|c|c|c|c|c|}
\hline Sex & & & $3.45(1.13-10.51)$ & 8.21 \\
\hline Female & 80 & 9 & & \\
\hline Male $^{\mathrm{d}}$ & 18 & 7 & & \\
\hline \multicolumn{5}{|c|}{ Gestational age at birth } \\
\hline Preterm $^{\mathrm{d}}$ & 12 & 0 & - & - \\
\hline Term & 86 & 16 & & \\
\hline
\end{tabular}

Type of delivery

$\begin{array}{lcc}\text { Cesarean }^{\mathrm{d}} & 76 & 9 \\ \text { Vaginal } & 22 & 7\end{array}$

\section{Fetal presentation}

$\begin{array}{lcc}\text { Breech }^{\mathrm{d}} & 10 & 0 \\ \text { Cephalic } & 88 & 16\end{array}$

\section{Amniotic fluid volume}

$16.92(0.88-322.36)$

$\begin{array}{lcc}\text { Normal } & 96 & 13 \\ \text { Oligohydramnios }^{\mathrm{d}} & 2 & 3\end{array}$

\section{Order of children}

$0.23(0.09-0.56)$

0.29

$\begin{array}{lcc}\text { First child } & 61 & 4 \\ \text { Second child } & 34 & 8 \\ \text { Third child or next children } & 3 & 4\end{array}$

\section{Family history of DDH}

$\begin{array}{lcc}\text { Positive } & 14 & 2 \\ \text { Negative }^{\mathrm{d}} & 84 & 13\end{array}$

Limb anomalies

$7.69(2.31-25.59)$

$5.02(0.99-25.42)$

$\begin{array}{lcc}\text { Negative } & 91 & 9 \\ \text { Positive }^{\mathrm{d}} & 7 & 7\end{array}$

\section{Renal abnormality}

$\begin{array}{lcc}\text { Negative } & 98 & 13 \\ \text { Positive }^{\mathrm{d}} & 0 & 3\end{array}$

Abbreviations: CI, confidence Interval; DDH, developmental dysplasia of the hip; OR, odds ratio.

${ }^{a}$ Values are expressed as median (range).

bimple logistic regression.

${ }^{\mathrm{c}}$ Multiple logistic regression (there were all variables in the model).

${ }^{\mathrm{d}}$ Reference category. 


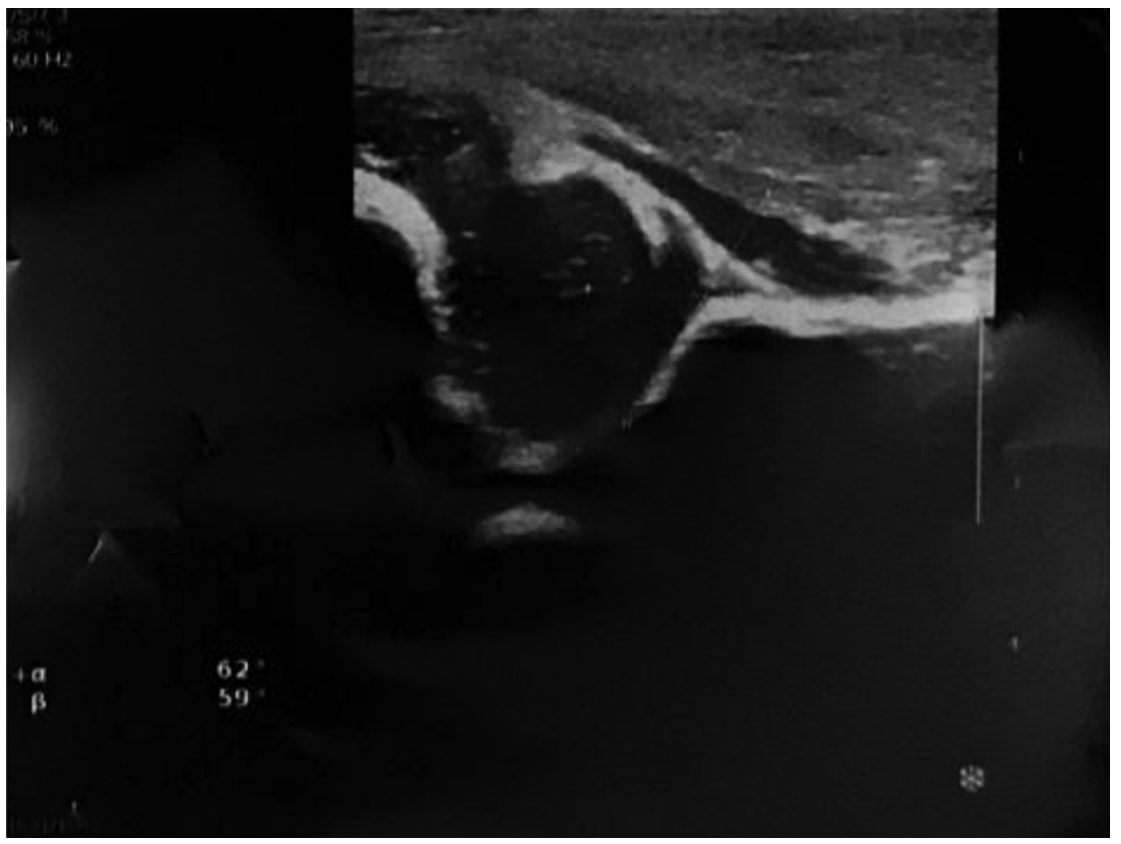

Figure 5. Eleven-week infant. The same patients in Figures 3 after 8 weeks of Pavlik cast shows complete recovery to type $\mathrm{I}($ alpha $=62$ and beta $=59)$.

\section{Discussion}

In the current study, 74 infants (114 abnormal hip joints) were examined. Overall, there was a significant correlation between the severity of involvement according to the Graf criteria, male gender, higher birth order, oligohydramnios, and renal and limb anomalies and poor response. Most of the findings were compatible with those of the previous studies. However, in some results, differences were noticed which could be explained. For instance, the success rate was $45.5 \%$ in a study by Al-Essa et al. (9), which was lower than that of the current study in which 98 (86\%) joints showed complete recovery. They treated $68.1 \%$ of infants younger than six months using the Pavlik harness orthosis, which is the first-line treatment in this age group, and the closed reduction was applied to $78 \%$ of older infants. Open reduction was only used for infants older than 18 months (9). This difference in the success rate can be attributed to the target population. In their study, all DDH cases had dislocation or dysplasia, while in the current study, most of the hip joints were type 2 a, all of which showed good response.

Likewise, the current study revealed that DDH, by $56.2 \%$, was more common in female patients. Studies showed that female cases are more prone to DDH due to estrogen effect (3). During follow-up, among the subjects that responded to treatment, $81.6 \%$ were female and $18.4 \%$ were male. The response rate was significantly higher in female patients in contrast with the results obtained by Omeroglu and Koparal (10), in which no association was found between gender and treatment response.

The average alpha angles on the first ultrasound in the responsive and non-responsive groups were 54.02 and 43.25 degrees and for beta angles, they were 66.38 and 80 degrees, respectively. Analysis of data showed that a decrease of alpha angle, which implies much severe DDH, significantly decreased treatment response. These findings were compatible with those of other studies, including White et al. (11), in which femoral head location in the inferior labrum was remarkably associated with better response, and a more superior or lateral location of the femoral head to the labrum was associated with treatment failure.

In the current study, the mean ages of the responsive and non-responsive groups were 6.32 and 7.88 weeks, respectively. Although the mean age of infants who were not treated successfully was higher, there was no significant correlation between age and treatment response. This difference can be attributed to the lower mean age in the current study, which was $6.54 \pm 6.6$ weeks. It can also be explained by the fact that patients older than 12 months were not included.

Follow-up length was 10 weeks in infants with positive 
family history, and 8.5 weeks in those without a family history. This finding may suggest that positive family history encourages families to attend more follow-up sessions. Overall, $87.5 \%$ of infants with family history and $86.5 \%$ of those without family history recovered. As a result, family history had no significant effect on the response rate. Omeroglu et al. examined the effect of variables including age, gender, side of involvement, family history, and other factors on response rate in infants with DDH. The results showed that side of involvement, family history, type of delivery, fetal presentation, and oligohydramnios had no significant correlation with response rate (10-12).

In conclusion, factors including the severity of hip involvement based on Graf criteria, male gender, higher birth order, oligohydramnios and renal and limb anomalies had a significant negative effect on treatment response. Although these risk factors seem to not change treatment choices, their presence could imply a more resistant DDH, which may encourage considering further treatment at an earlier stage. Overall, the success rate in the treatment of DDH is high which and for this reason, early detection and treatment of DDH is strongly suggested.

\section{Footnotes}

Authors' Contributions: Study concept and design: MM, HA, and AZ. Acquisition of data: MM, HA, and AZ. Analysis and interpretation of data: AZ and NB. Drafting of the manuscript: NB Critical revision of the manuscript for important intellectual content: NB and MM. Statistical analysis: AZ, HA, and MM. Administrative, technical, and material support: MM, AZ, HA, and NB. Study supervision: MM.

Clinical Trial Registration Code: The clinical trial registration code was IR.TUMS.MEDICINE.REC.1395.1928.a.

Conflict of Interests: There was no conflict of interest in our study.

Ethical Approval: The ethical approval was obtained from the Children's Hospital Center Hospital.

Funding/Support: There was no funding or supporting source in our study.

\section{References}

1. Synder M, Niedzielski K, Grzegorzewski A. Ultrasound of hip joint in newborns and infants. Ortop Traumatol Rehabil. 2003;5(6):717-21. [PubMed: 18034063]

2. Roof AC, Jinguji TM, White KK. Musculoskeletal screening: developmental dysplasia of the hip. Pediatr Ann. 2013;42(11):229-35. doi: 10.3928/00904481-20131022-10. [PubMed: 24168117].

3. Akman A, Korkmaz A, Aksoy MC, Yazici M, Yurdakok M, Tekinalp G. Evaluation of risk factors in developmental dysplasia of the hip: results of infantile hip ultrasonography. Turk J Pediatr. 2007;49(3):2904. [PubMed: 17990583].

4. Vafaee AR, Baghdadi T, Baghdadi A, Jamnani RK. DDH epidemiology revisited: Do we need new strategies? Arch Bone Jt Surg. 2017;5(6):4402.

5. Arti H, Mehdinasab SA, Arti S. Comparing results of clinical versus ultrasonographic examination in developmental dysplasia of hip. $J$ Res Med Sci. 2013;18(12):1051-5. [PubMed: 24523795]. [PubMed Central: PMC3908525].

6. Sllamniku S, Bytyqi C, Murtezani A, Haxhija EQ. Correlation between avascular necrosis and the presence of the ossific nucleus when treating developmental dysplasia of the hip. J Child Orthop. 2013;7(6):501-5 doi: 10.1007/s11832-013-0538-z. [PubMed: 24432113]. [PubMed Central: PMC3886353].

7. Guarniero R. Dysplasia of hip development: Update. Rev Bras Ortop. 2010;45(2):116-21. doi: 10.1016/S2255-4971(15)30279-2. [PubMed: 27022528]. [PubMed Central: PMC4799082].

8. Barakat AS, Zein AB, Arafa AS, Azab MA, Reda W, Hegazy MM, et al. Closed reduction with or without adductor tenotomy for developmental dysplasia of the hip presenting at walking age. Curr Orthop Pract. 2017;28(2):195-9. doi: 10.1097/BCO.0000000000000478. [PubMed: 28286603]. [PubMed Central: PMC5325246].

9. Al-Essa RS, Aljahdali FH, Alkhilaiwi RM, Philip W, Jawadi AH, Khoshhal KI. Diagnosis and treatment of developmental dysplasia of the hip: A current practice of paediatric orthopaedic surgeons. J Orthop Surg (Hong Kong). 2017;25(2):2.3094990177172E+15. doi: 10.1177/2309499017717197. [PubMed: 28659058].

10. Omeroglu H, Koparal S. The role of clinical examination and risk factors in the diagnosis of developmental dysplasia of the hip: A prospective study in 188 referred young infants. Arch Orthop Trauma Surg. 2001;121(1-2):7-11. doi:10.1007/s004020000186. [PubMed: 11195123].

11. White KK, Sucato DJ, Agrawal S, Browne R. Ultrasonographic findings in hips with a positive Ortolani sign and their relationship to Pavlik harness failure. J Bone Joint Surg Am. 2010;92(1):113-20. doi: 10.2106/JBJS.H.01880. [PubMed: 20048103].

12. Omeroglu $\mathrm{H}$. Use of ultrasonography in developmental dysplasia of the hip. J Child Orthop. 2014;8(2):105-13. doi: 10.1007/s11832-014-0561-8. [PubMed: 24510434]. [PubMed Central: PMC3965765] 